\title{
DRUG DIFFUSION TRANSPORT THROUGH HUMAN SKIN
}

\author{
A. WALICKA* \\ University of Zielona Góra, Faculty of Mechanical Engineering \\ ul. Szafrana 4, 65-516 Zielona Góra, POLAND \\ E-mail: A.Walicka@ijame.uz.zgora.pl \\ B. IWANOWSKA-CHOMIAK \\ University Hospital of Zielona Góra, Oncology Department \\ ul. Zyty 26, 65-046 Zielona Góra, POLAND \\ E-mail: B.Chomiak@op.pl
}

\begin{abstract}
The stratum corneum (SC) forms the outermost layer of the human skin and is essentially a multilamellar lipid milieu punctuated by protein-filled corneocytes that augment membrane integrity and significantly increase membrane tortuosity. The lipophilic character of the SC, coupled with its intrinsic tortuosity, ensure that it almost always provides the principal barrier to the entry of drug molecules into the organism. Drugs can be administered either as suspensions or as solutions and the formulation can range in complexity from a gel or and ointment to a multilayer transdermal path. In this paper, we discuss theoretical principles used to describe transdermal release and we show that relatively simple membrane transport models based on the appropriate solution to the Fick's second law of diffusion can be used to explain drug release kinetics into such a complex biological membrane as the human skin. To apply the Fick's law we introduced into our considerations a brick-and-mortar model with two factors of tortuosity. Assuming that the mortar thickness is variable we also introduced the hindrance factor allowing us to model this variability. Having the modified Fick's equation we presented its general solution and two special cases of this solution frequently applicable in permeation experiments. It seems that the solutions presented herein better approximate the real conditions of drug delivery then these well known.
\end{abstract}

Key words: drug delivery, human skin, diffusion.

\section{Introduction}

Fluid flow and diffusion transport within natural tissues play an important role in morphogenesis, metabolism functions and pathogenesis. In the design of new biomaterials mimicking biological tissues, it is well recognized today that a three-dimensional "in vitro" culture better recapitulates physiological cell environment. Fluid flow and solid strain that are imposed within tissue not only affect cell nourishment but also exert on cell mechanical actions such as pressure effects, drag interactions and viscous shear stresses.

Every cell is distinguished from its environment by means of a semipermeable membrane. This membrane plays a crucial role in the ability of the cell to function; the loss of membrane integrity, except for brief periods, would almost certainly lead to the cell's death.

This paper addresses modelling and treatment of mass transport through biological tissues, specially the skin, considered as a porous medium; the mass transport is narrowed down to the diffusion in the skin drug delivery.

The skin drug delivery can be subdivided into topical and transdermal. In a topical delivery the drug is intended to act at skin level, this is indicated for the treatment of skin diseases.

\footnotetext{
* To whom correspondence should be addressed
} 
The aim of transdermal delivery is getting a systemic release and in this case the skin represents a porous barrier that allows drug penetration [1]. The transdermal drug delivery is a viable administration route for potent, not only low-molecular weight therapeutic agents, which has to be precise in the control of drug distribution. This action is specially recommended for many drugs that are difficult to be taken since they must be delivered slowly over a prolonged period to have a beneficial effect $[1,2]$.

The transdermal drug administration has several interesting advantages over other systemic administration routes such as [2-6]:

- reduction of first-pass drug degradation as the liver is initially bypassed,

- reduction of over dosage peaks that can appear in oral administration,

- existence of variable delivery conditions typical of the gastrointestinal tract.

Most often in the therapy of local and external changes, antibiotics, antifungal drugs, antivirals, glucocorticoids, anti-inflammatory drugs and analgesics, chemotherapeutics, in aesthetic medicine botulinum toxin in the treatment of hyperhidrosis, are administrated transdermally. Systemic effects are characterized by transdermal systems of analgesics and hormonal drugs $[5,6]$.

One of the oldest drug administration applied since the 1950, acting topically on the skin and systemically, is hydrocortisone, while transdermal administration was applied a bit later [7].

The simplest transdermal formulation consists of a semi-solid vehicle, e.g., an ointment or a cream containing a finely divided suspension of the drug. The control of the drug delivery was first described by Higuchi [8,9] and next developed by Michaels et al. [10] and by Bunge [11]. Some theoretical considerations, including the solutions to the Fick's diffusion equation, were presented by Hadgraft [12] and Guy and Hadgraft [13]. Many recent theoretical considerations on the drug diffusion transport through the skin are based on their solutions [14-21].

Motivated by the aforementioned papers, the principle idea of this work is to present a new mathematical model of the skin porosity. The recent ("classical") model of the skin porosity uses a brickand-mortar model with uniform thickness of the mortar (Fig.2). We would like to propose some modification to this model assuming variable thickness of the mortar (which is closer to the reality) and introducing the so called "hindrance factor" [22].

\section{Skin as a porous biomembrane}

The skin being the body's first defense against infection should not be treated - in general - as a homogenous medium. Even in a very basic description, human skin in made of multiple constituent composite layers (Milington and Wilkinson, [23]), each providing specific function with varying thickness. A schematic representation of human skin is shown in Fig.1.

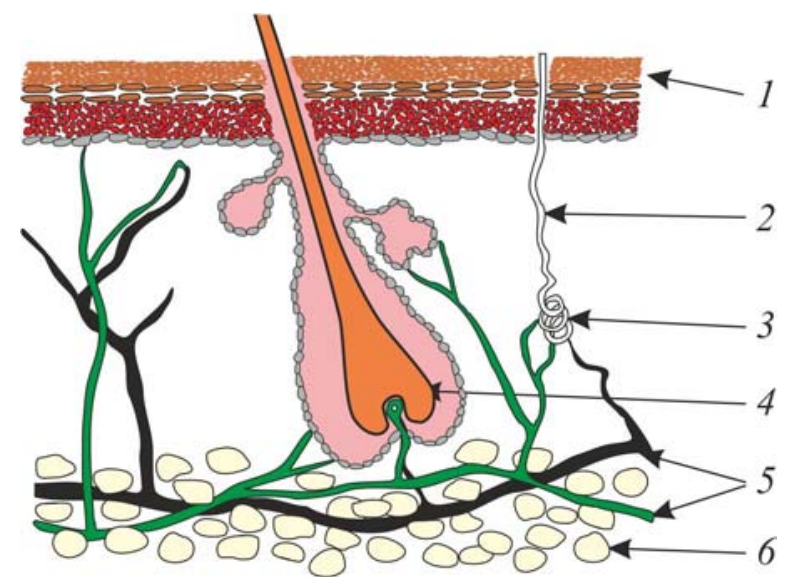

Fig.1. Schematic representation of the skin; 1 - stratum corneum, 2 - sweat duct, 3 - sweat gland, 4 - hair follicle, 5 - blood vessels, 6 - fat lobule. 
The outer skin layer, the epidermis, is without vasculature and acts as a protective barrier preventing molecular transport. The epidermis is a foundation of viable living cells being a transition to flat dead cells at its outer surface. The thin outermost layer of the epidermis is called the "stratum corneum" (SC) and it is most resistive to drug transport through the skin.

Below the epidermis there is a highly vascular inner skin layer, the dermis, that is characterized by a the large network of capillaries with high blood flow rates exceeding several times the metabolic requirements. The primary reason of such high perfusion rates in the dermis is to regulate body temperature. The dermis is also characterized by high collagen content, that provides the skin with its structural support.

Below the dermis there lies a thermally insulative fatty layer that also acts to conserve body heat. The skin, at most sites on the body, is perforated by appendageal pathways in the form of sweat glands and hair follicles.

The SC is composed of 15-20 layers of corneocytes, that is flat dead cell shells, which are interconnected by a lipid lamellar bilayer structure in a crystallinegel phase. Transport circumventing the barrier function of the SC is primarily associated to occur within the lamellar lipid structure of the SC (Bouwstra et al. [24]; Madison [25]). The thin SC corneocytes-lipid porous matrix (Fig.2) may be conceptualized as a brick-andmortar structure in which the highly impermeable corneocyte cells are represented by the brick and the slightly less impermeable intercellular lipid lamellar bilayers are represented by the mortar.

a)

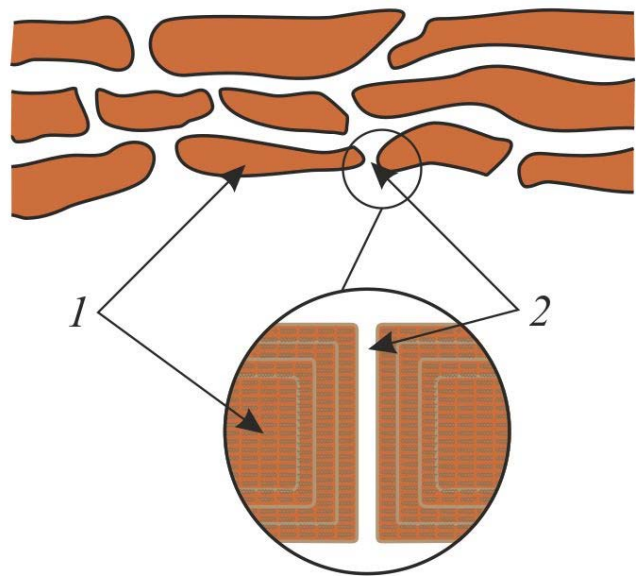

b)

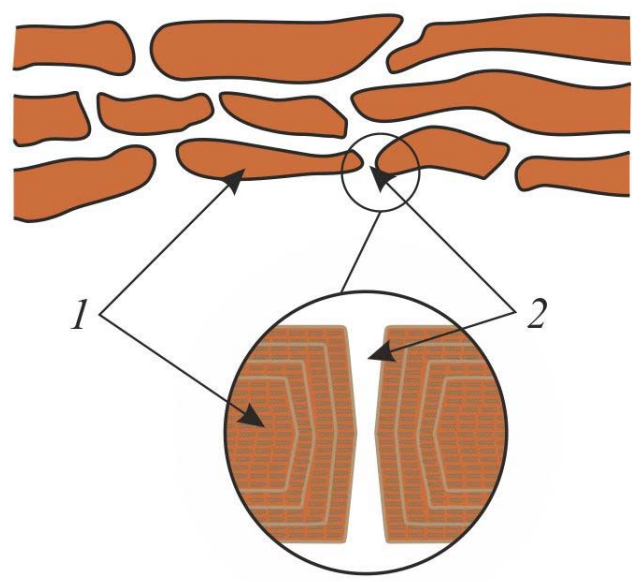

Fig.2. Schematic representation of the stratum corneum (SC); 1 - corneocyte, 2 - lipid bilayers, a) scheme hitherto accepted, b) scheme just now proposed.

Traditional drug deliveries often result in low efficacy and unintentional treatment of healthy tissues. Oral intake may result in drug denaturation in the gastro-intestinal track; drugs consisting of water soluble molecules may degrade before reaching intended locations of treatment. Injection of drugs into the circulatory system may result in lower selectivity level of the drug's region influence. Transdermal drug delivery is one method that allows localized delivery by transporting the drug directly through the skin to the desired target, as it is necessary, for example, in chemotherapy for drugs with a high toxicity.

\section{Diffusion through brick-and-mortar structure}

The representation of the SC matrix consisting of a symmetric brick-and-mortar model was introduced at first by Michaels et al. [10] in which the interstitial horizontal lengths between vertical spaces are all of the same length (Fig.3a). A nonsymmetric variation of this model was introduced by Johnson et al. [14]; this model describes more accurately the lateral diffusive path by using an offset, $\omega$, that is defined as the ratio of long, $d_{L}$, to short, $d_{S}$, lateral diffusion path lengths (Fig.3.b). This offset is equal to $\omega=d_{L} / d_{S}$; for the symmetric model the offset is equal to $\omega=1$. 

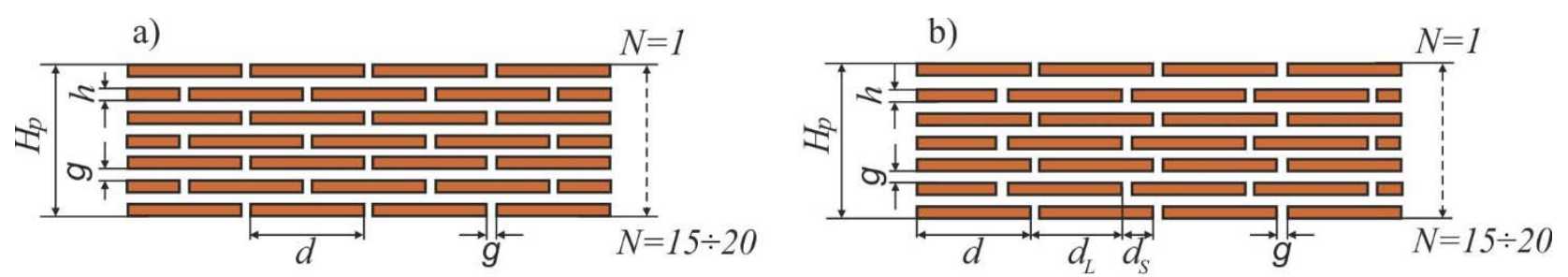

Fig.3. Brick-and-mortar representations of the SC matrix: a) symmetric, b) nonsymmetric.

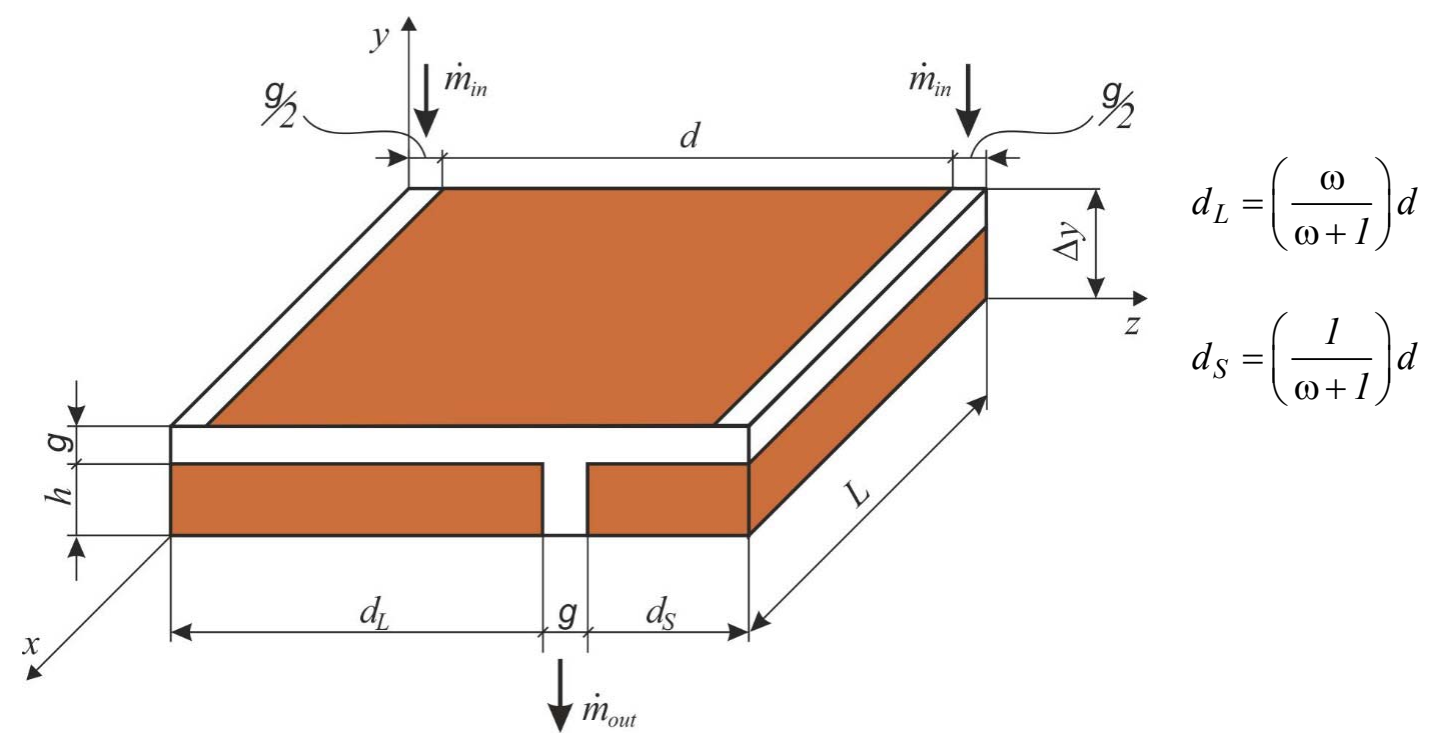

Fig.4. Schematic view of a slice of the SC matrix.

Figure 4 shows one slice of the SC matrix; here $\dot{m}_{\text {in }}$ and $\dot{m}_{\text {out }}$ are, respectively, the drug mass flow rates into and out of the slice of the SC. Assuming that mass transport is restricted to the intercellular regions of the SC and following Kushner et al. [26] (see also [21]) we may write the mass balance for the flowing permeant (drug solute) in the form

$$
\frac{1}{\tau_{v}} \frac{\partial F(y, t)}{\partial y}=\varphi_{p} \frac{\partial C(y, t)}{\partial t}
$$

where

$$
\begin{aligned}
& \tau_{v}=\frac{d+g+h}{g+h} \text { is the volume tortuosity factor, } \\
& \varphi_{p}=\frac{g}{d+g} \text { is the porosity of the } \mathrm{SC}, \\
& F(y, t) \text { is the mass flux, } \\
& C(y, t) \text { is the drug concentration. }
\end{aligned}
$$

Taking into account that the real mortars (intercellular regions of the SC) are not regular let us introduce the so called "hindrance factor" (Walicka et al. [22]) considering the irregularity of the mortar. Then Eq.(3.1) takes the form 


$$
\frac{\psi}{\tau_{v}} \frac{\partial F(y, t)}{\partial y}=\varphi_{p} \frac{\partial C(y, t)}{\partial t}
$$

where $\psi$ is the hindrance factor.

Note that some mathematical considerations on the flows of Newtonian and non-Newtonian fluids through irregular capillary tubes and fissures one may find in [27, 28].

Consider now the diffusive mass flow rate of the drug delivered through the intercellular regions of the SC; according to Johnson et al. [14] it may be evaluated as (see also [21])

$$
F(y, t)=\frac{\varphi_{p} D}{\tau_{f}} \frac{\partial C(y, t)}{\partial y} .
$$

or, after introducing the hindrance factor

$$
F(y, t)=\frac{\varphi_{p} \psi}{\tau_{f}} \frac{\partial C(y, t)}{\partial y} .
$$

where

$$
\begin{aligned}
& D \text { is the diffusion coefficient, } \\
& \tau_{f}=\frac{d_{l a t}+g+h}{g+h} \text { is the flux tortuosity factor, } \\
& \frac{1}{d_{\text {lat }}}=\frac{1}{d_{l}}+\frac{1}{d_{s}}
\end{aligned}
$$

or

$$
d_{\text {lat }}=\frac{\omega}{(1+\omega)^{2}} d \text { is the lateral diffusion path length. }
$$

Now substituting Eq.(3.4) into Eq.(3.2) yields the final form of the Fick second law of diffusion through the irregular intercellular regions of the SC

$$
\frac{d C}{d t}=\frac{\psi^{2} D}{\tau_{v} \tau_{f}} \frac{\partial^{2} C}{\partial y^{2}}
$$

or in a more general form

$$
\frac{\partial C}{\partial t}+(\bar{v} \nabla) C=\frac{\psi^{2} D}{\tau_{v} \tau_{f}} \frac{\partial^{2} C}{\partial y^{2}},
$$

where $\bar{v}$ is the velocity vector of the flow in the SC intercellular regions.

One of more general forms of this equation proposed by Picioreanu et al. [29] is as follows

$$
\frac{\partial C}{\partial t}+(\bar{v} \nabla) C=\operatorname{div}(\varepsilon D \operatorname{grad} C)+\Phi,
$$


where $\varepsilon$ is the coefficient of concentration and $\Phi$ is the source field of diffusion.

Different variants of this equation have been discussed by many researchers, except early cited also by DiMicco and Sah [30], Barta and Maroudas [31], Klein and Sah [32], who discussed diffusion and drug transport in a human articular cartilage.

Note that in the case of the absence of flow $(\overline{\mathrm{v}}=0)$, Eq.(3.6) takes the form of Eq.(3.5).

The factors $\tau_{v}$ and $\tau_{f}$ are given for one lipid layer; in the case of $N$ corneocyte layers and of $(N-1)$ intercellular lateral lipid layers in the SC, the expressions for $\tau_{v}$ and $\tau_{f}$ are given by Kushner et al. [26]

$$
\begin{aligned}
& \tau_{v}= \frac{(N-1) d+N h+(N-1) g}{N h+(N-1) g}, \\
& \tau_{f}=\frac{(N-1) \frac{\omega}{(1+\omega)^{2}} d+N h+(N-1) g}{N h+(N+1) g} .
\end{aligned}
$$

The hindrance factor for human skin takes the values from the bracket $0.8 \leq \psi \leq 1$.

\section{Diffusion transport through the stratum corneum}

Taking into account the fact that the flow in the brick-and-mortar fissure is a one-dimensional flow we may rewrite Eq.(3.6) in the following form

$$
\frac{\partial C}{\partial t}+|\overline{\mathrm{v}}| \frac{\partial C}{\partial y}=\frac{\psi^{2} D}{\tau_{v} \tau_{f}} \frac{\partial^{2} C}{\partial y^{2}} .
$$

This equation should be solved for the general case with the following boundary and initial conditions

$$
C(y=0, t)=C_{1}, \quad C\left(y=H_{p}, t\right)=C_{2}, \quad C(y, t=0)=C_{0},
$$

where $C_{1}$ is the bulk concentration on the receiver side of the SC, $C_{2}$ is the bulk concentration on the donor side of the SC and $C_{0}$ is the initial concentration of the drug within the intercellular lipids of the SC. Note that $y=0$ corresponds to the outlet of the stratum corneum, whereas $y=H_{p}$ corresponds to the inlet of the stratum corneum.

Introducing the non-dimensional magnitudes

$$
\tilde{C}=\frac{C}{C_{2}}, \quad \tilde{y}=\frac{y}{H_{p}}, \quad \alpha=\frac{|\overline{\mathrm{v}}| H_{p} \tau_{v} \tau_{f}}{\psi^{2} D}, \quad \tilde{t}=\frac{\psi^{2} D}{\tau_{v} \tau_{f} H_{p}^{2}} t,
$$

one may rewrite Eq.(4.1) as follows

$$
\frac{\partial \tilde{C}}{\partial \tilde{t}}+\alpha \frac{\partial \tilde{C}}{\partial \tilde{y}}=\frac{\partial^{2} \tilde{C}}{\partial \tilde{y}^{2}}
$$




\subsection{Steady-state diffusion through the SC}

First, let us consider the steady-state diffusion through the SC. The Fick's equation (4.4) for the steady-state case is as follows

$$
\frac{d^{2} \tilde{C}}{d \tilde{y}^{2}}=\alpha \frac{d \tilde{C}}{d \tilde{y}}
$$

Solving this equation with the boundary conditions (4.2), one obtains

$$
\tilde{C}=\tilde{C}_{1}+\frac{\tilde{C}_{2}-\tilde{C}_{1}}{1-e^{\alpha}}\left(1-e^{\alpha \tilde{y}}\right)
$$

or

$$
C=C_{1}+\frac{C_{2}-C_{1}}{1-e^{\alpha}}\left(1-e^{\alpha \frac{y}{H_{p}}}\right) .
$$

Note that for $\alpha \rightarrow 0$, there is

$$
\tilde{C}=\tilde{C}_{1}+\left(\tilde{C}_{2}-\tilde{C}_{1}\right) \tilde{y},
$$

or

$$
C=C_{1}+\left(C_{2}-C_{1}\right) \frac{y}{H_{p}}
$$

therefore the diffusion distribution is linear.

\subsection{Non-steady state diffusion through the SC}

Let us rewrite Eq.(4.4) as follows

$$
\frac{\partial \tilde{C}}{\partial \tilde{t}}=\frac{\partial^{2} \tilde{C}}{\partial \tilde{y}^{2}}-\alpha \frac{\partial \tilde{C}}{\partial \tilde{y}} .
$$

A standard method of obtaining the solution of a partial differential equation of second order is to assume that the variables are separable (Kacki [33]; Crank [34]). Applying this method (called the method of separation of variables) one obtains the following solution [21]

$$
\begin{aligned}
& \tilde{C}=\tilde{C}_{1}+\frac{\tilde{C}_{2}-\tilde{C}_{1}}{1-e^{\alpha}}\left(1-e^{\alpha \tilde{y}}\right)+\sum_{m=1}^{\infty} A_{m} \sin (m \pi \tilde{y}) \exp \left(\frac{\alpha}{2} \tilde{y}-\lambda_{m}^{2} \tilde{t}\right)+ \\
& +\tilde{C}_{0} \sum_{m=1}^{\infty} \frac{8 m \pi}{\alpha^{2}+4(m \pi)^{2}}\left(e^{-\frac{\alpha}{2}} \cos (m \pi)-1\right) \sin (m \pi \tilde{y}) \exp \left(\frac{\alpha}{2} \tilde{y}-\lambda_{m}^{2} \tilde{t}\right),
\end{aligned}
$$

where 


$$
\begin{aligned}
& A_{m}=\frac{8 m \pi}{\alpha^{2}+4(m \pi)^{2}}\left[\left(\tilde{C}_{1}+\frac{\tilde{C}_{2}-\tilde{C}_{1}}{1-e^{\alpha}}\right)\left(e^{-\frac{\alpha}{2}} \cos (m \pi)-1\right)-\frac{\tilde{C}_{2}-\tilde{C}_{1}}{1-e^{\alpha}}\left(e^{\frac{\alpha}{2}} \cos (m \pi)-1\right)\right], \\
& \lambda_{m}^{2}=\frac{1}{4}\left[\alpha^{2}+4(m \pi)^{2}\right] .
\end{aligned}
$$

Note that for $\alpha \rightarrow 0, A_{m}$ tends towards

$$
A_{m}=\frac{2}{m \pi}\left[\tilde{C}_{2} \cos (m \pi)-\tilde{C}_{1}\right]
$$

and the solution given by Eq.(4.9) approaches the form (Crank [34])

$$
\begin{aligned}
& \tilde{C}=\tilde{C}_{1}+\left(\tilde{C}_{2}-\tilde{C}_{1}\right) \tilde{y}+\frac{2}{\pi} \sum_{m=1}^{\infty} \frac{\tilde{C}_{2} \cos (m \pi)-\tilde{C}_{1}}{m} \sin (m \pi \tilde{y}) \exp \left(-\lambda_{m}^{2} \tilde{t}\right)+ \\
& +\frac{4 \tilde{C}_{0}}{\pi} \sum_{m=0}^{\infty} \frac{\sin (2 m+1) \pi \tilde{y}}{2 m+1} \exp \left(-\lambda_{2 m+1}^{2} \tilde{t}\right),
\end{aligned}
$$

where

$$
\lambda_{m}^{2}=(m \pi)^{2}, \quad \lambda_{2 m+1}^{2}=[(2 m+1) \pi]^{2}
$$

\subsection{Mass transport in non-steady state diffusion}

The total amount of mass per unit area, $M(t)$, delivered across the SC is given by a time integral over the flux $F(y, t)$

$$
M(t)=\int_{0}^{t} F(y=0, t) d t=\frac{\varphi_{p} \psi}{\tau_{f}} \int_{0}^{t}\left[D \frac{\partial C(y, t)}{\partial y}\right]_{y=0} d t
$$

Substitution of the dimensional form of Eq.(4.12) and integration over time yields

$$
\begin{aligned}
& M(t)=\frac{\varphi_{p} \psi D t}{\tau_{f} H_{p}}\left(C_{2}-C_{1}\right)+\frac{2 \varphi_{p} \psi \tau_{v} H_{p}}{\pi^{2}} \sum_{m=1}^{\infty} \frac{C_{2} \cos (m \pi)-C_{1}}{m^{2}}\left[1-\exp \left(-\frac{\psi^{2} D m^{2} \pi^{2} t}{\tau_{v} \tau_{f} H_{p}^{2}}\right)\right]+ \\
& +\frac{4 \varphi_{p} \psi C_{0} \tau_{v} H_{p}}{\pi^{2}} \sum_{m=0}^{\infty} \frac{1}{(2 m+1)^{2}}\left[1-\exp \left(-\frac{\psi^{2} D(2 m+1)^{2} \pi^{2} t}{\tau_{v} \tau_{f} H_{p}^{2}}\right)\right] .
\end{aligned}
$$

For the special case when $C_{1}=C_{0}=0$, which is frequently applicable to permeation experiments, Eq.(4.15) can be further simplified

$$
M(t)=\frac{\varphi_{p} \tau_{v} H_{p} C_{2}}{6 \psi}\left\{\frac{6 \psi^{2} D t}{\tau_{v} \tau_{f} H_{p}^{2}}-1-\frac{12}{\pi^{2}} \sum_{m=1}^{\infty} \frac{(-1)^{m}}{m^{2}} \exp \left(-\frac{\psi^{2} D m^{2} \pi^{2} t}{\tau_{v} \tau_{f} H_{p}^{2}}\right)\right\} .
$$


Here it was assumed that (Korn and Korn, [35])

$$
\cos (m \pi)=(-1)^{m}, \quad \sum_{m=1}^{\infty} \frac{(-1)^{m}}{m^{2}}=-\frac{\pi^{2}}{12}
$$

Equation (4.16) describes mass transport across a rate-controlling membrane and it is identical with the equation describing diffusion across the SC in situations where drug release from a transdermal drug delivery system is rapid and transport across the stratum corneum is the rate-limiting step before fast entry into the viable epidermis. At the long time values, the exponential terms tend to zero and the full solution can be approximated by

$$
M(t)=\frac{\varphi_{p} \tau_{v} H_{p} C_{2}}{\psi}\left(\frac{\psi^{2} D t}{\tau_{v} \tau_{f} H_{p}^{2}}-\frac{1}{6}\right) .
$$

The shortest lag time describing the time necessary to reach steady-state diffusion is essential for the drug delivery through the skin. This time can be evaluated by setting $M(t)=0$ in Eq.(4.17), which yields

$$
t_{\text {lag }}=\frac{\tau_{v} \tau_{f} H_{p}^{2}}{6 \psi^{2} D}
$$

When the time $t$ reaches the value $\left(t=t_{\text {lag }}\right)$, the diffusion may be described by Eqs (4.7). This case of drug release represents the so called "lag effect".

A different drug release called "burst effect" arises when $C_{1}=0, C_{2}=C_{0}$, then the expression describing drug release is given by

$$
M(t)=\frac{\varphi_{p} \tau_{v} H_{p} C_{2}}{6 \psi}\left\{\frac{6 \psi^{2} D t}{\tau_{v} \tau_{f} H_{p}^{2}}+2+\frac{12}{\pi^{2}} \sum_{m=1}^{\infty} \frac{1}{m^{2}} \exp \left(-\frac{\psi^{2} D m^{2} \pi^{2} t}{\tau_{v} \tau_{f} H_{p}^{2}}\right)\right\} .
$$

At the long time values there is

$$
M(t)=\frac{\varphi_{p} \tau_{v} H_{p} C_{2}}{\psi}\left(\frac{\psi^{2} D t}{\tau_{v} \tau_{f} H_{p}^{2}}+\frac{1}{3}\right)
$$

Having general solution (4.12) of the Fick's equation it is possible to find other particular solutions for different boundary and initial conditions. These solutions may be found in the literature. Note that most of these solutions consider only straight mortar fissures without their tortuosity. Any of these solutions does not make allowance for variable cross section (variable thickness) of the mortar fissures.

\section{Conclusions}

In the paper, we have focussed on analytical solutions of the Fick's equation describing mass diffusion through the porous membrane. These solutions have the advantage that the terms in the equations can be clearly related to physical parameters within the drug transport system. Some analytical solutions 
have the drawback of being limited to systems having relatively simple geometries and boundary conditions. On the other hand, it seems that numerical methods are considerably more powerful and can be used to model more complex situations which more closely resemble those encountered experimentally [34]. More complex mathematical models that represent release into a biologically realistic membrane, e.g., taking into account the structural heterogeneity, and which incorporate diffusion in three-dimensions and any timedependent variation of the boundary conditions will have to employ advanced analytical methods of solutions.

The aim of this paper was to improve the analytical method using a brick-and-mortar model, with uniform thickness of the mortar, describing the porosity of the stratum corneum. This aim was achieved by two steps dependent on:

- the introduction of two tortuosity factors,

- the introduction of the hindrance factor.

The influence of tortuosity on mass transport in porous media is well known but in drug diffusion transport tortuosity is rarely taken into account.

The hindrance factor represents the influence of variable thickness of the mortar fissure on the mass transport in porous medium. Introduction of this factor allows us to consider the fissure variability in a simple way.

It seems that the proposed methods of solution will contribute to a broader use of the analytical methods for a better understanding of drug diffusion transport through the skin.

\section{Nomenclature}

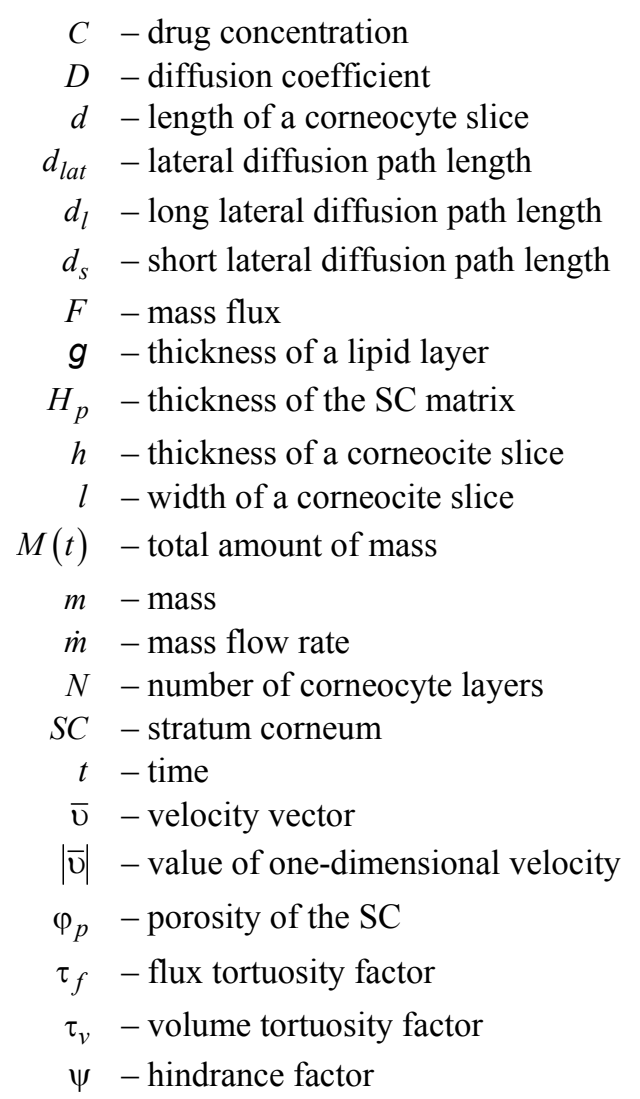




\section{References}

[1] Kalia Y.N. and Guy R.H. (2001): Modeling transdermal drug release. - Adv. Drug Delivery Rev., vol.48, pp.159172.

[2] Carreras N., Alonso C., Marti M. and Lis M.J. (2015): Mass transport model through the skin by microencapsulation system. - J. Microencapsulation, vol.32, No.4, pp.358-363.

[3] Cal K. (2009): Across skin barrier; known methods, new performances. - In: Frontiers in Drug Design and Discovery, vol.4 (Caldwell G.W., ur-Rahman A., Yan Z., Choudhary M.J., Eds), pp.162-188.

[4] Cal K. and Stefanowska J. (2010): Methods for skin permeation enhancement of drug substances. - Technology of drug form (in Polish: Technologia postaci leku), vol.66, No.7, pp.514-520.

[5] Glogau R.G. (2007): Topically applied botulinum toxin type A for the treatment of primary axillary hyperhidrosis: results of randomized, blinded, vehicle-controlled study. - Dermatol. Surg., vol.33, pp.76-80.

[6] Prausnitz M.R. and Langer R. (2008): Transdermal drug delivery. - Nat. Biotechnol., vol.26, No.11, pp.1261-1268.

[7] Ahluwalic A. (1998): Topical glucocorticosteroids and the skin-mechanism of action. - Med. Inflam., vol.7, pp.183193.

[8] Higuchi T. (1960): Physical chemical analysis of percutaneous absorption process from creams and ointments. - J. Soc. Cosmet. Chem., vol.11, pp.85-97.

[9] Higuchi T. (1961): Rate of release of medicaments from ointment basis containing drugs in suspension. - J. Pharm. Sci., vol.50, pp.874-875.

[10] Michaels A.S., Chandraskeran S.K. and Shaw J.E. (1975): Drug permeation through human skin: theory and in vitro experimental measurement. - Amer. Inst. Chem. Eng., vol.21, No.5, pp.985-996.

[11] Bunge A.L. (1998): Release rate from topical formulations containing drugs in suspension. - J. Control. Rel., vol.52, No.1, pp.141-148.

[12] Hadgraft J. (1979): Calculations of drug release rates from controlled release devices. The slab. - Int. J. Pharmaceutics, vol.2, pp.177-194.

[13] Guy R.H. and Hadgraft J. (1980): A theoretical description relating skin penetration to the thickness of the applied medicament. - Int. J. Pharmaceutics, vol.6, pp.321-332.

[14] Johnson M.E., Blankshtein D. and Langer R. (1997): Evaluation of solute permeation through the stratum corneum: lateral bilayer diffusion as the primary mechanism. - J. Pharm. Sci., vol.86, No.10, pp.1162-1172.

[15] Ouriemchi E.M. and Vernaud J.M. (2000): Processes of drug transfer with three different polymeric systems with transdermal drug delivery. - Comp. Theor. Polymer Sci., vol.10, pp.391-401.

[16] Coceani N., Colombo I. and Grassi M. (2003): Acyclovir permeation through rat skin: mathematical modelling and vitro experiments. - Int. J. Pharmaceutics, vol.254, pp.197-210.

[17] Yamashita F. and Hashida M. (2003): Mechanistic and empirical modeling of skin permeation of drugs. - Adv. Drug Delivery Rev., vol.55, pp.1185-1199.

[18] He N., Waner K.S., Higuchi W.I. and Li K. (2005): Model analysis of flux enhancement across hairless mouse skin induced by chemical permeation enhancers. - Int. J. Pharmaceutics, vol.297, pp.9-21.

[19] Herkenne C., Naik A., Kalia Y.N., Hadgraft J. and Guy R. (2008): Effect of propylene glycol on ibuprofen absorption into human skin in vivo. - J. Pharm. Sci., vol.97, No.1, pp.185-197.

[20] Grassi M. (2008): Membranes in drug delivery. - In: Handbook of Membrane Separations: Chemical, Pharmaceutical, Food and Biotechnological Applications (Pabby A.K., Rizvi S.S.H. and Sastre A.M., Eds), CRC Press, New York, pp.427-464.

[21] Walicka A. (2017): Rheology of Fluids in Mechanical Engineering. - Zielona Góra: University Press.

[22] Walicka A., Walicki E., Jurczak P. and Falicki J. (2018): Effect of hindrance factors on a squeeze film of a porous bearing with a DeHaven fluid. - Submited to: Machine Dynamics Research. 
[23] Milington P.F. and Wilkinson R. (1983): Skin. - Cambridge University Press.

[24] Bouwstra J.A., Honeywell-Nguyen P.L., Gooris G.S. and Ponec M. (2003): Structure of the skin barrier and its modulation by vesticular formulations. - Progress in Lipid Research, vol.42, No.1, pp.1-36.

[25] Madison K.C. (2003): Barrier function of the skin: , la raison d'Être" of the epidermis. - Journal of Investigative Dermatology, vol.121, No.2, pp.231-241.

[26] Kushner J., Deen W.: Blankshtein D. and Langer R. (2007): First-principles, structure-based transdermal transport model to evaluate lipid partition and diffusion coefficients of hydrophobic permeants soley from stratum corneum permeation experiments. - J. Pharm. Sci., vol.96, No.12, pp.3236-3251.

[27] Walicka A. (2018a): Simulation of the flow through porous layer composed of converging-diverging capillary fissures and tubes. - Int. J. Appl. Mech. Eng., vol.23, No.1, pp.161-185.

[28] Walicka A. (2018b): Flows of Newtonian and power-law fluids in symmetrically corrugated fissures and tubes. Int. J. Appl. Mech. Eng., vol.23, No.1, pp.187-215.

[29] Picioreanu C., van Loosdrecht M.C.M. and Heijnen J.J. (2001): Two-dimensional model of biofilm detachment caused by internal stress from liquid flow. - Biotech. Bioeng., vol.72, No.2, pp.205-218.

[30] DiMicco M.A. and Sah R.L. (2003): Dependence of cartilage matrix composition on biosynthesis, diffusion and reaction. - Transport in Porous Media, vol.50, pp. 57-73.

[31] Barta E. and Maroudas A. (2006): A theoretical study of the distribution of insulin-like growth factor in human articular cartilage. - J. Theor. Biology, vol.241, pp.628-638.

[32] Klein T.J. and Sah R.L. (2007): Modulation of depth-dependent properties in tissue-engineered cartilage with a semi-permeable membrane and perfusion: a continuum model of matrix metabolism and transport. - Biomech. Model. Mechanobiology, vol.6, pp.21-23.

[33] Kącki E. (1967): Thermokinetics (in Polish). -Warszawa: WN-T.

[34] Crank J. (1975): The Mathematic of Diffusion. - $2^{\text {nd }}$ Edition, Oxford: Clarendon Press.

[35] Korn G.A. and Korn T.M. (2013): Mathematical Handbook for Scientists and Engineers. - New York: Dover Publication, Inc.

Received: February 12, 2018

Revised: April 20, 2018 